\title{
INJURY TO THE MAJOR AIRWAYS DURING SUBTOTAL ESOPHAGECTOMY: INCIDENCE, MANAGEMENT, AND SEQUELAE
}

Jan B. F. Hulscher, MD

Ester ter Hofstede, $\mathrm{MD}^{\mathrm{a}}$

Jaap Kloek, MD

Hugo Obertop, MDa

Peter de Haan, MD

J. Jan B. van Lanschot, MD
Objective: The objective of this study was to gain insight into the incidence and sequelae of injury to the major airways during subtotal esophagectomy.

Methods: We performed an analysis of 383 consecutive patients undergoing this procedure between 1993 and 1999. Indications were adenocarcinoma (220), squamous cell carcinoma (121), and other (42). Transhiatal resection was done in 269 (70\%) patients and transthoracic resection in 114 (30\%).

Results: There were 4 men and 2 women (median age 57 years; range 45 to 68 years) with injury to the major airways, recognized during surgery in 5 patients and on the first postoperative day in the other. Five lesions occurred during transhiatal resection $(5$ of $269=1.8 \%$ ) and 1 during transthoracic resection ( 1 of $114=0.8 \% ; P=.67$ ). The injury occurred proximal to the carina in 5 patients and in the left main bronchus in the other. All injuries could be closed primarily. The defect was covered with pericardium in 1 patient and with pleura in 2 patients. In all cases the gastric tube was placed over the defect. Pulmonary complications developed in 4 patients. Patients with tracheal injury required artificial ventilation for a longer period (median 6 days vs 1 day; $P=.02$ ) and stayed longer in the intensive care unit (median 11 vs 3 days; $P<.01$ ) than patients without such injury, although hospital time was not significantly prolonged (median 23 vs 16 days; $P=.09)$. There was no associated mortality.

Conclusion: Tracheobronchial injury is a rare complication of subtotal esophagectomy. It can be managed effectively by primary closure and apposition of vital tissue (gastric tube) to the defect. It is associated with pulmonary complications, leading to prolonged assisted ventilation and stay in the intensive care unit, but mortality is rare. ( $\mathrm{J}$ Thorac Cardiovasc Surg 2000;120:1093-6)
E sophagectomy can be divided into the transthoracic E approach and the transhiatal approach. The former is a combined abdominothoracocervical approach through which the esophagus and adjacent tissues are resected

From the Departments of Surgery, ${ }^{\mathrm{a}}$ Cardio-pulmonary Surgery, ${ }^{\mathrm{b}}$ and Anesthesiology, ${ }^{\mathrm{c}}$ Academic Medical Center at the University of Amsterdam, Amsterdam, The Netherlands.

Received for publication Feb 28, 2000; revisions requested April 13, 2000; revisions received May 2, 2000; accepted for publication July 14, 2000.

Address for reprints: J. B. F. Hulscher, MD, Academic Medical Center, Department of Surgery, Suite G4-134, Meibergdreef 9, 1105 AZ Amsterdam, The Netherlands (E-mail: J.B.Hulscher@AMC.UVA.NL).

Copyright () 2000 by The American Association for Thoracic Surgery

$0022-5223 / 2000 \$ 12.00+0 \quad \mathbf{1 2 / 1 / 1 1 0 1 8 2}$

doi: $10.1067 / \mathrm{mtc} .2000 .110182$ and lymphadenectomy can be performed. The latter is an abdominocervical resection (without thoracotomy), in which the esophagus is mobilized through the surgically widened hiatus of the diaphragm and resected (stripped) distalward. Often no formal lymphadenectomy is done.

Opponents of the transhiatal resection suggest that it not only is oncologically unwarranted but also can be potentially dangerous, especially when the tumor is situated proximal to the carina. The esophagus lies in close relation with trachea and main stem bronchi at one side and the vertebral column and the aorta at the other. Blunt resection of the esophagus could result in damage to these vital structures, with devastating results.

However, although these complications are feared, their incidence is low. The incidence of tracheal tears 
during transhiatal resection is around 1\%; in experienced hands this number might even be lower. ${ }^{1}$ There are almost no data regarding the incidence of injury to the trachea or main stem bronchi during transthoracic resections or its sequelae. We analyzed the incidence of injury to the major airways during both transhiatal and transthoracic esophageal resections. The associated morbidity and mortality will also be reported.

\section{Patients and methods}

An analysis was performed of the 383 consecutive patients who underwent subtotal esophagectomy between July 1993 and August 1999. The analysis was based on prospectively collected data from a database. Transhiatal resections were carried out in 269 (70\%) patients; the other 114 (30\%) patients underwent transthoracic resection. Indications were high-grade dysplasia $(n=12)$, adenocarcinoma $(n=220)$, squamous cell carcinoma $(n=121)$, and other, including nonmalignant causes $(n=30)$. For tumors located distal to the carina, the transhiatal approach was used preferably, just as for nonmalignant causes and high-grade dysplasia. There were 100 patients with tumors located at or proximal to the carina, 52 of whom underwent transthoracic resection. Sixtytwo patients with distal adenocarcinoma participating in a randomized trial were allocated to a transthoracic resection with 2-field lymph node dissection.

Statistical analysis was performed with the aid of the SPSS package (SPSS, Inc, Chicago, Ill). The $\chi^{2}$ and Mann-Whitney $U$ tests were applied as appropriate for categoric and continuous data follow-up.

\section{Results}

There were 4 men and 2 women, with a median age of 57 years (range 45-68 years), with injury to the major airways. In 5 patients injury to the major airways was noticed during the operation, and in 1 additional patient it was noticed in the intensive care unit (ICU) on the first postoperative day after urgent reintubation for autodetubation. Three patients had a squamous cell carcinoma, 2 had adenocarcinoma, and 1 patient had high-grade dysplasia. In 2 cases the carcinoma was located proximal to the carina. There were $4 \mathrm{~T} 3$ tumors and $1 \mathrm{~T} 1 \mathrm{(sm})$ tumor. One patient with a squamous cell carcinoma underwent preoperative chemotherapy. Radiation therapy had not been administered.

Five lesions occurred during transhiatal resection $(5 / 269=1.8 \%)$, whereas 1 lesion occurred during transthoracic resection $(1 / 114=0.8 \%)$. This difference is not statistically significant $(P=.67)$. Also when the tumors proximal to or at the carina are analyzed separately, there is no difference between transhiatal or transthoracic approaches. In 5 cases the injury occurred proximal from the carina, and in 1 it was located in the left main bronchus. There was no correlation between tumor location and location of the tear. Three patients underwent a microscopically radical $\mathrm{R} 0$ resection; microscopic and macroscopic tumor was left behind in 1 patient each. There was no correlation between the occurrence of injury to the major airways and tumor stage (including $\mathrm{T}$ and $\mathrm{N}$ stage).

The air leak was noticed immediately during the operation in 5 patients. In 4 patients undergoing transhiatal resection it was noticed by large amounts of air escaping through the hiatus and a pressure drop in the circuits of the anesthesiologist. In 2 patients the leak occurred during stripping of the esophagus, whereas it occurred during the transhiatal dissection prior to stripping in the other 2 patients. In the patient undergoing transthoracic resection the tumor was attached to the left main bronchus, which was torn accidently during the dissection; this was noticed immediately under direct vision. After immediate insertion of a doublelumen tube to gain control of the airway a conversion to a right-sided thoracotomy was performed (during transhiatal resection). In the patient undergoing transthoracic resection, the double-lumen tube could be replaced distal to the defect, providing adequate ventilation. In 1 patient the air leak was noticed after urgent reintubation for autoextubation, with air escaping through the cervical wound.

All lesions could be closed primarily with 5-0 Vicryl stitches (Ethicon, Inc, Somerville, NJ). The defect was subsequently covered with pericardium in 1 case and with pleura in 2 cases. In all patients the gastric tube was placed against the closed defect posterior to the trachea and loosely attached with a few stitches. Care was taken that the longitudinal staple line of the gastric tube was not in direct contact with the airway closure.

Postoperatively, 4 of the 6 patients had pulmonary complications such as pneumonia and/or atelectasis (vs 72 of 377 or $19.0 \%$ in the patients without injury to the major airways; $P=.009, \chi^{2}$ ). This is also reflected in a prolonged time requiring artificial ventilation and in the ICU (Table I). Hospital time was also prolonged in patients with injury to the major airways, but this did not reach statistical significance.

On follow-up, patients did well functionally without symptoms of stricture formation. No routine bronchoscopic examination was done on follow-up. After a median follow-up of 1.2 years ( 2 months -2.5 years), 4 patients have had recurrent disease, 3 of whom have died.

\section{Discussion}

Injury to the major airways is an uncommon complication of esophagectomy $(6 / 363=1.6 \%)$, which can be 
managed adequately by primary closure and apposition of vital tissue (gastric tube) to the defect. Although all patients had significant pulmonary morbidity, they all recovered.

Opponents of transhiatal resection suggest that tracheal lesions occur more often in the transhiatal resection because of the close relation between the esophagus and trachea. Blind stripping of the esophagus might lead to damage to the major airways. Usually tracheal lesions are vertical tears in the membranous posterior portion of the trachea. In the largest series published on transhiatal resections, Orringer and colleagues ${ }^{1}$ reported an incidence of $1 \%$ for tracheal lesions in patients undergoing transhiatal esophagectomy, with an inverse relation between incidence of tracheal lesions and surgical experience. When the literature of the past decade is reviewed, the incidence of tracheal tear is $0.6 \%$ after transhiatal resection, whereas it has not been mentioned after transthoracic resection. ${ }^{2}$ However, most authors do not mention this complication, especially when describing the results of transthoracic resection. ${ }^{2}$

Careful selection of patients for transhiatal resection is mandatory to prevent injury to the tracheobronchial tree. In our institution, tumors at or proximal to the carina are preferably approached transthoracically because of the position of the esophagus between the trachea, vertebral column, and aorta. However, when the general health of the patient precludes thoracotomy, a transhiatal resection is performed. In patients with a proximal tumor, bronchoscopy should be performed before a (transthoracic) resection takes place to exclude ingrowth in the mucosa. Although this was not administered in the present series, neoadjuvant radiation therapy might make dissection more difficult.

Transhiatal resection of distal esophageal cancers is not as blunt/blind a resection as has often been described by its opponents. The tumor can be mobilized through the widened hiatus of the diaphragm, with dissection under direct vision until well proximal to the tumor. Only the normal part of the esophagus is therefore resected bluntly. However, peritumoral inflammatory adhesions may develop, making dissection and stripping more dangerous, which might have been the cause of the airway lesion in some of our patients with distal tumors. During the procedure, the pressure of the dissecting finger should always be on the esophagus to prevent pressure on the posterior membranous trachea, especially over the bulb of the endotracheal tube.

Tracheal lesion is a very uncommon complication that is thought to result in an immediate inability to
Table I. Time spent on artificial ventilation, in the ICU, and in the hospital (days; median, range) of patients with and without injury to the tracheobronchial tree after subtotal esophagectomy

\begin{tabular}{lccc}
\hline & No injury & Injury & P value $^{*}$ \\
\hline Ventilation time & $1(0-33)$ & $6(1-40)$ & .02 \\
Time in ICU & $3(1-71)$ & $13(3-47)$ & $<.01$ \\
Hospital time & $16(9-155)$ & $23(14-58)$ & .11
\end{tabular}

*Mann-Whitney $U$ test.

ventilate the patient. In addition, life-threatening respiratory complications might ensue in the postoperative course. In this series, 4 of 6 patients had respiratory complications, probably because of initial hypoventilation in combination with the aspiration of blood through the defect, especially during the expiration phase in which airway pressures are low, leading to atelectasis and pneumonia. Although most patients in this series had respiratory complications, required artificial ventilation for a significantly longer time, and spent more time in the ICU, there was no associated mortality. All patients were able to leave the hospital in relatively good condition.

Treatment of these injuries should be straightforward. When an air leak is suspected perioperatively, either by the surgeon noticing large amounts of air escaping through the operative field or by the anesthetist noticing a sharp decrease in pressure in the circuits or large changes in the capnography readings, immediate control of the airway is mandatory. After insertion of a double-lumen tube, conversion to a thoracotomy can take place in the case of a transhiatal resection. Care has to be taken not to enlarge the defect during the insertion of the double-lumen tube. During a transhiatal resection the defect may be covered by the surgeon's finger, thus guiding the ventilation tube. In the case of a transhiatal resection, we position the patient in the dorsal decubitus position with the aid of a beanbag, so that immediate conversion can take place without having to reposition or to redrape the patient. ${ }^{3}$ After conversion to a thoracotomy, primary repair can be carried out. When possible, a regular tube may be reinserted with the balloon distal to the defect. Airway pressures should be kept as low as possible, and early extubation should be strived for. When prolonged ventilation is necessary, the double-lumen tube may be changed for a regular tube, which should be inserted under bronchoscopic guidance.

Postoperatively, tracheal injury might be suggested by persistent air leak through the chest tubes or subcutaneous emphysema developing over the face and neck. 
Bronchoscopic examination will subsequently reveal the lesion. When the air leak is noticed postoperatively, as occurred once in this series, the patient should undergo reoperation unless the lung is fully expanded, the patient remains well oxygenated, and the gastric tube already abuts the lesion completely. Negative suction might be applied to the chest tubes to try to expand the lung, but when this is not successful operative repair should take place. ${ }^{4}$ Intensive lung physiotherapy and bronchial toileting are mandatory, and broad-spectrum antibiotics are to be considered.

The defect can be closed primarily followed by the apposition of vital tissue to the defect. Primary repair is said to carry a high risk of subsequent stricture formation, but this did not occur in the present series. Stricture formation might occur when primary repair narrows the lumen or can only be performed under tension. In those cases, patches from pleura or pericardium may be used, but these patches are relatively poorly vascularized and sometimes not available because of tumor ingrowth. Also, postoperative radiation might cause damage to the graft site. ${ }^{5}$ The use of polytetrafluoroethylene* or Marlex grafts (Bard Implants, Billerica, Mass) has also been described, but this is less attractive because it leads to the presence of a foreign body in a possibly infected area. ${ }^{4,6}$ The gastric tube is a relatively well-vascularized structure that can be positioned over the defect without adding substantial tension. Placement of the gastric tube with its attached omentum adds vital tissue to the defect, improving vascular-

\footnotetext{
*Gore-Tex graft, registered trademark of W. L. Gore \& Associates, Inc, Flagstaff, Ariz.
}

ization and thereby increasing local resistance to infection. The use of pedunculated muscle flaps (intercostal or pectoral) is probably not indicated in the acute treatment of these injuries, but it might be an important treatment option for long-standing tracheal injuries associated with mediastinitis, filling the dead space with well-vascularized and highly resistant tissue.

\section{Conclusion}

Injury to the major airways is a rare complication of both transhiatal and transthoracic esophagectomy. Careful patient selection and meticulous surgical technique should prevent many of these injuries. When they occur, primary repair followed by apposition of the gastric tube to the defect is usually sufficient. Pulmonary morbidity is high, but mediastinitis and mortality are rare.

\section{REFERENCES}

1. Orringer MB, Marshall B, Iannettoni MD. Transhiatal esophagectomy: clinical experience and refinements. Ann Surg 1999;230:392-403.

2. Hulscher JBF, Tijssen JGP, Obertop H, Van Lanschot JJB. Transthoracic versus transhiatal resection for carcinoma of the esophagus: a meta-analysis. Ann Thorac Surg. In press.

3. De Graaf PW, Idenburg FJ, Obertop H. A uniform approach for esophagectomy with or without thoracotomy. Surg Gynecol Obstet 1991;173:149-52.

4. Kannan RR, Mahajan V. Tracheal injury during transhiatal mobilization of the esophagus. J Surg Oncol 1999;71:186-8.

5. Gorenstein LA, Abel JG, Patterson GA. Pericardial repair of a tracheal laceration during transhiatal esophagectomy. Ann Thorac Surg 1992;54:784-6.

6. Millikan KW, Pytynia KB. Repair of tracheal defect with GoreTex graft during resection of carcinoma of the esophagus. J Surg Oncol 1997;66:134-7. 